\title{
'Hidden mounts' in Duchenne muscular dystrophy
}

\author{
C. J. Suresh Chandran \\ Department of Neurology, Kerala Institute of Medical Sciences, Trivandrum, Kerala, India
}

A ten-year-old boy presented with inability to run, progressive difficulty in walking and weakness of proximal muscles of lower and upper limbs from the age of three years. By the time he presented to us, he was unable to stand even with support. Neurological examination revealed hypertrophy of supraspinatus, infraspinatus, deltoid, lattismus dorsi, rhomboids and calf muscles, bilaterally. 'Valley sign' was present [Figure 1]. Serum CPK was 8000 U/L. EMG showed myopathic potentials. Polymerase chain reaction identified dystrophin gene deletion mutation and diagnosis of Duchenne muscular dystrophy (DMD) was confirmed. Abdominal CT cuts were taken to evaluate for abdominal muscles. CT abdomen showed hypertrophy of abdominal muscles, paraspinal muscles and psoas bilaterally-'hidden mounts' [Figure 2].

Calf muscles are the commonest muscles to undergo hypertrophy in DMD, followed by infraspinatus. But various other muscle groups also show enlargement in DMD. Hypertrophy of abdominal and paraspinal muscles are probably overlooked. Our patient had hypertrophic abdominal muscles, paraspinal muscles and psoas bilaterally as demonstrated in the abdominal CT- the 'hidden mounts'. Valley sign is a specific sign of DMD with $90 \%$ sensitivity. It is due to the wasting of muscles in the posterior axillary fold (valley). On either side of this valley there are two mounts -hypertrophic infraspinatus inferomedially and deltoid hypertrophy superolaterally. The whole appearance is like a 'valley between two mounts'. ${ }^{[1]}$

\section{Reference}

1. Pradhan S. New clinical sign in Duchenne muscular dystrophy. Pediatr Neurol 1994;11:298-300.

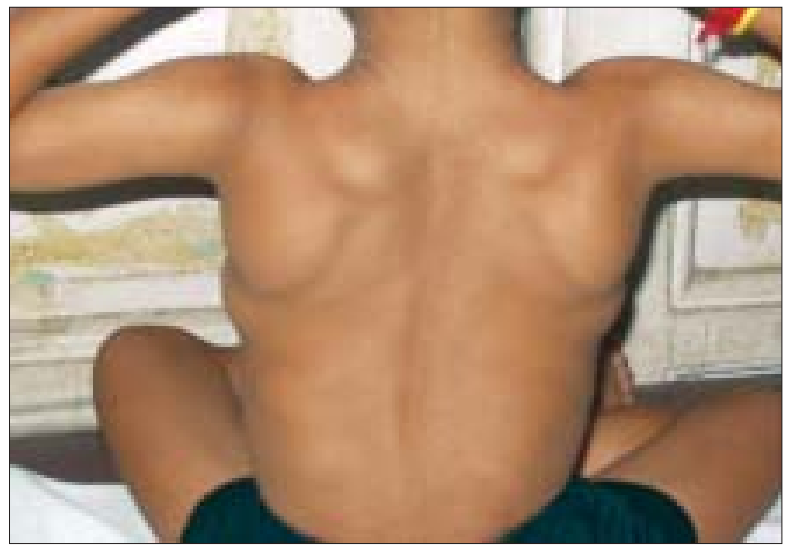

Figure 1: Valley sign. Also note the hypertrophy of supraspinatus, rhomboids and lattismus dorsi

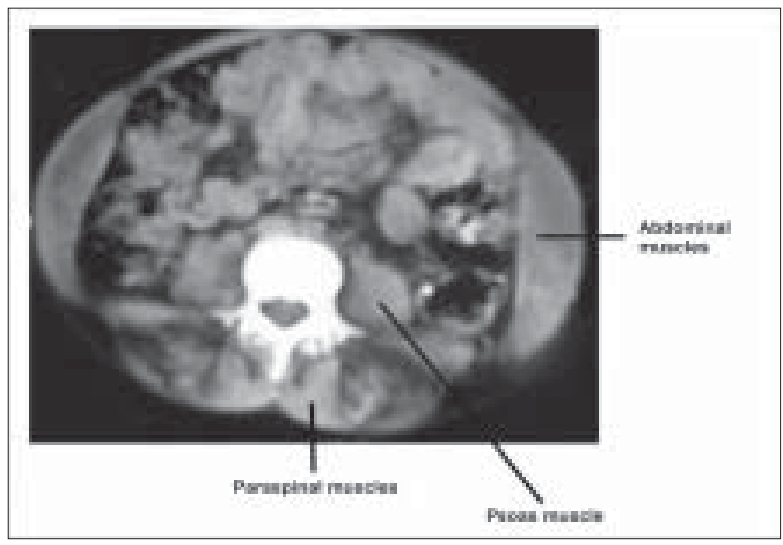

Figure 2: CT abdomen showing hypertrophy of abdominal muscles, paraspinal muscles and psoas bilaterally-the 'Hidden mounts'

Accepted on 04-09-2008

Source of Support: Nil, Conflict of Interest: None declared. 